\section{A replication of a non-linear association of educational attainment and suicide rates among the elderly using five-year data}

A recent cross-national study demonstrated that the relationship between elderly suicide rates and educational attainment is curvilinear (U-shaped curve) (Shah and Chatterjee, 2008). That study used only one-year data on suicide rates for the latest available year. These findings may have been spurious as suicide rates can randomly fluctuate year on year (Shah and Coupe, 2009). Therefore, this curvilinear relationship was examined using a oneyear average of five-year data on elderly suicide rates to establish the accuracy of the earlier findings.

Data on elderly suicide rates for males and females in the age-bands $65-74$ years and $75+$ years for the latest five years were ascertained from the World Health Organization (WHO) (www.who.int/ whosis/database/mort/table1.cfm). The median (range) of the latest available year for the 83 different countries was 2005 (1983-2007); this data set was more recent than that used in the previous study (Shah and Chatterjee, 2008). The one-year average suicide rate was calculated by dividing the sum of the suicide rates for each of the five years by 5 . Data on the education index were obtained from the United Nations Development Program (www.hdr.undp.org/hdr2006/pdfs/ report/HDR_2006_Tables.pdf) for the year 2000 for these 83 countries. The education index is a composite measure of the adult literacy rate and the combined gross enrollment ratios for primary, secondary and tertiary schools. It was used as a proxy marker for educational attainment. It is scored on a scale of 0 to 1 , with higher scores reflecting greater degree of educational attainment.
Curve estimation regression model was used to test the curvilinear relationship (U-shaped curve) fitting the quadratic equation $y=a+b x+c x^{2}$ (where $y$ is the suicide rate for elderly people, $x$ is the education index, and $a, b$ and $c$ are constants).

The characteristics of curve estimation regression models are illustrated in Table 1 . The relationship of suicide rates for both sexes in both elderly age-bands with the education index rates was curvilinear (a U-shaped curve) and fitted the above quadratic equation.

This study, using a one-year average of five years of data on elderly suicide rates, successfully replicated the findings of the earlier study using only one-year data on elderly suicide rates. These findings therefore suggest that the curvilinear (Ushaped) relationship between elderly suicide rates and educational attainment is robust and accurate. It was important to establish the accuracy and robustness of earlier findings before developing studies to identify potential explanations for the curvilinear relationship.

\section{References}

Shah, A. K. and Chaterjee, S. (2008). Is there a relationship between elderly suicide rates and educational attainment? A cross-national study. Aging and Mental Health, 12, 795-799.

Shah, A. K. and Coupe, J. (2009). A comparative study of elderly suicides in England and Wales, Scotland and Northern Ireland: trends over time and age-associated trends. International Psychogeriatrics, 21, 581-587.
AJIT SHAH
Professor of Ageing, Ethnicity and Mental Health, University of Central Lancashire, Preston and Consultant Psychiatrist, West London Mental Health NHS Trust, London, U.K.
Email: ajit.shah@wlmht.nhs.uk

Table 1. Curve regression models for the relationship between elderly suicide rates and educational attainment

\begin{tabular}{|c|c|c|c|c|}
\hline & $\mathrm{R}^{2}$ & $\mathrm{~F}$ & $P$ & REGRESSION EQUATION \\
\hline Males aged $65-74$ years & 0.23 & 11.68 & $<0.001$ & $y=136.49-431.03 x+339.96 x^{2}$ \\
\hline Males aged $75+$ years & 0.19 & 9.37 & $<0.001$ & $y=107.26-392.90 x+348.03 x^{2}$ \\
\hline Females aged $65-74$ years & 0.20 & 9.90 & $<0.001$ & $y=2.42-37.05 x+46.05 x^{2}$ \\
\hline Females aged $75+$ years & 0.13 & 6.14 & $<0.005$ & $y=-45.72+58.6 x+3.12 x^{2}$ \\
\hline
\end{tabular}

$y=$ elderly suicide rate.

$x=$ education index.

Degrees of freedom $=80$. 